\title{
T cell circuits that sense antigen density with an ultrasensitive threshold
}

\section{Hernandez-Lopez, Rogelio A.}

2021-03-12

\begin{abstract}
Hernandez-Lopez , R A , Yu , W , Cabral , K A , Creasey , O A , Lopez Pazmino , M D P , Tonai , Y, De Guzman , A, Mäkelä , A , Saksela , K, Gartner , Z J \& Lim , W A 2021 , ' T cell circuits that sense antigen density with an ultrasensitive threshold ' , Science , vol. 371 , no. 6534 , pp. 1166-+ . https://doi.org/10.1126/science.abc1855
\end{abstract}

http://hdl.handle.net/10138/330717

https://doi.org/10.1126/science.abc1855

publishedVersion

Downloaded from Helda, University of Helsinki institutional repository.

This is an electronic reprint of the original article.

This reprint may differ from the original in pagination and typographic detail.

Please cite the original version. 


\section{SYNTHETIC BIOLOGY}

\section{T cell circuits that sense antigen density with an ultrasensitive threshold}

\author{
Rogelio A. Hernandez-Lopez ${ }^{1,2}$, Wei Yư ${ }^{1}$, Katelyn A. Cabral ${ }^{2,3,4 *}$, Olivia A. Creasey ${ }^{2,3,4 *}$, \\ Maria del Pilar Lopez Pazmino ${ }^{1,2}$, Yurie Tonai ${ }^{1}$, Arsenia De Guzman ${ }^{1}$, Anna Mäkelä ${ }^{5}$, Kalle Saksela ${ }^{5}$, \\ Zev J. Gartner ${ }^{2,3}$, Wendell A. Lim ${ }^{1,2} \dagger$
}

Overexpressed tumor-associated antigens [for example, epidermal growth factor receptor (EGFR) and human epidermal growth factor receptor 2 (HER2)] are attractive targets for therapeutic T cells, but toxic "offtumor" cross-reaction with normal tissues that express low levels of target antigen can occur with chimeric antigen receptor (CAR)-T cells. Inspired by natural ultrasensitive response circuits, we engineered a two-step positive-feedback circuit that allows human cytotoxic T cells to discriminate targets on the basis of a sigmoidal antigen-density threshold. In this circuit, a low-affinity synthetic Notch receptor for HER2 controls the expression of a high-affinity CAR for HER2. Increasing HER2 density thus has cooperative effects on T cells-it increases both CAR expression and activation-leading to a sigmoidal response. T cells with this circuit show sharp discrimination between target cells expressing normal amounts of HER2 and cancer cells expressing 100 times as much HER2, both in vitro and in vivo.

T he specificity with which chimeric antigen receptor (CAR)-T cells can recognize and kill tumor cells and discriminate against normal cells remains limited (1-3). A major challenge is finding surface proteins that are absolutely tumor specific (4). CAR-T cells are effective in treating hematologic cancers (5-7), but they indiscriminately kill both cancerous and normal B cells because they target the lineage-specific protein CD19.
The loss of B cells is tolerable, but the killing of normal tissue when treating solid cancers with CAR-T cells remains a major challenge that can lead to toxicity and, in some cases, has proven lethal (8-10). Antigen receptors such as epidermal growth factor receptor (EGFR) and human epidermal growth factor receptor 2 (HER2) are overexpressed in cancers, but they are also expressed at lower densities in normal epithelial tissues (11, 12). Thus, anti-HER2 CAR-T cells have shown, in some cases, toxic crossreaction with normal organs (8). Such ontarget, off-tumor toxicity has been observed for CARs directed at several other overexpressed tumor-associated antigens $(13,14)$. Although some toxicity cases can be managed clinically (9), ideally, engineered T cells should reliably discriminate cancer cells from normal cells on the basis of antigen density (Fig. 1A, top).

To widen their therapeutic window, engineered $\mathrm{T}$ cells must sense target antigen density with a sigmoidal response and a sharper killing threshold (Fig. 1A, bottom). Dose-response behaviors in which small changes in input can generate large, nonlinear changes in output activity are referred to as "ultrasensitive" responses $(15,16)$.

Ultrasensitive behavior is observed in many critical regulatory systems and can be achieved through various mechanisms, ranging from allosteric molecules (for example, hemoglobin) to more complex regulatory cascades or circuits (17-19). Ultrasensitive circuits shift linear responses toward switch-like "all-or-none" responses in many biological systems $(15,18,20)$. In T cells, binding of the cytokine interleukin-2 (IL-2) to basally expressed low-affinity receptors results in induced expression of the highaffinity alpha subunit of the IL-2 receptor (CD25) (21). Thus, IL-2 acts on T cells in two ways: It both directly activates the cells and makes the cells more sensitive to IL-2, thereby
Fig. 1. Design of T cells with ultrasensitive antigendensity sensing. (A) Ideal therapeutic T cells will distinguish between tumor cells that express high antigen density and normal cells that express low antigen amounts. A CAR-T cell with a standard linear response curve distinguishes poorly between high- and low-density cells. Effective discrimination requires a sigmoidal ultrasensitive dose-response curve. (B) Design of two-step recognition circuit. A synNotch receptor detects an antigen (HER2) with low affinity. This synNotch receptor, when fully activated, induces expression of a high-affinity CAR. The low-affinity synNotch acts as a high-antigen-density filter, and the high-affinity CAR activates T cell killing and proliferation, acting as an amplifier. TF, transcription factor. (C) Densities of the tumor-associated antigen HER2 on engineered stable cell lines of human leukemia K562. Representative flow cytometry plots $(n=3)$ are shown. These cell lines can be compared to tumor cell lines (fig. S1A). The average HER2 molecules per cell was measured $(n=3)$ as shown in fig. S1A. To construct different HER2 sensing systems, we used a series of anti-HER2 scFvs with affinities spanning a 100-fold range. Ab, antibody; APC, allophycocyanin; AU, arbitrary units. (D) Binding affinities for anti-HER2 scFvs used in this study (for details of sequences and binding affinity measurements, see fig. S2). Biolayer interferometry sensograms show the binding kinetics for human HER2 and immobilized anti-HER2 scFvs. Data are shown as colored lines, and the best fit for data to a 1:1 binding model is shown in pink. HER2 concentrations used for binding affinity measurements are indicated. BLI, biolayer interferometry.
A

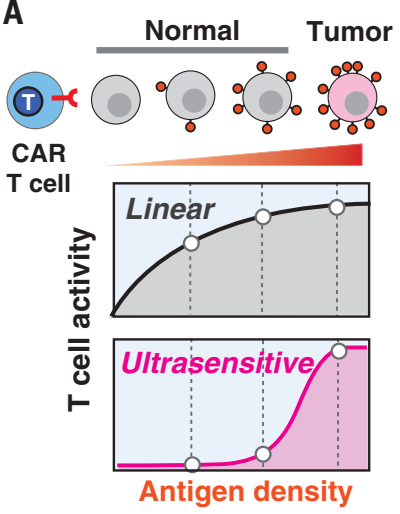

C

Engineered HER2 target cell lines (K562)

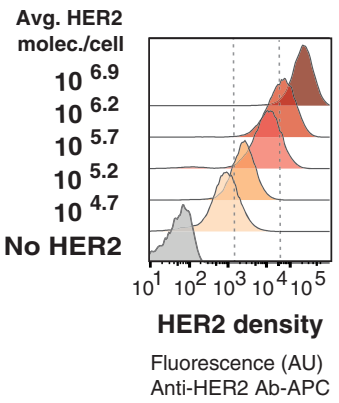

B

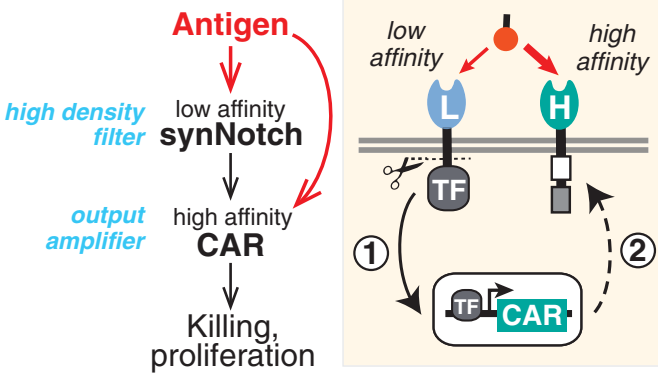

D Anti-HER2 single chain antibodies (scFv)
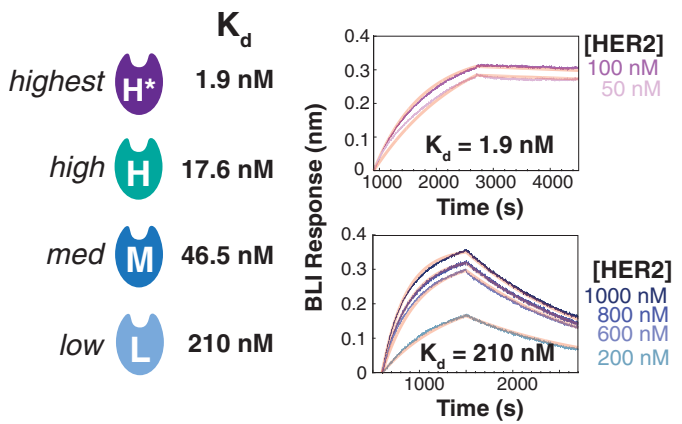
resulting in a form of positive feedback and cooperativity.

We used a modular approach to engineer ultrasensitive T cells (22-24). Our strategy recognizes the cognate antigen with a lowaffinity synthetic notch (synNotch) receptor, which would, in turn, induce the expression of a high-affinity CAR for the same antigen (synNotch receptors activate transcription of a genetically encoded payload when they engage input ligand) (Fig. 1B) (23-25). In this circuit design, the low-affinity synNotch receptor acts as a filter and constrains transcription induction to occur only when the $\mathrm{T}$ cell encounters target cells with high antigen expression. Once past this initial filter, the induced high-affinity CAR permits strong $\mathrm{T}$ cell killing and proliferation. Together, the circuit could yield an all-or-none, ultrasensitive response.

To evaluate $\mathrm{T}$ cell circuits engineered to achieve density-dependent recognition of the HER2 antigen, we constructed a series of stable human leukemia (K562) tumor cell lines that differ only in their amount of HER2 expression over a 100-fold range (Fig. 1C). The HER2 densities correspond to those of several cancer cell lines (fig. S1A). A clinically relevant goal is to be able to reliably discriminate between cells expressing $>10^{6.5}$ [HER2 pathology score of 3+, as defined by American Society of Clinical Oncology-College of American Pathologists (ASCO-CAP) scoring guidelines] versus $10^{4.5}$ molecules per cell (HER2 score of 0 or $1+$, termed "HER2 negative"), as these are the expression amounts found in several HER2-amplified cancers and several normal HER2-expressing human tissue samples (26), respectively. To build the synNotch receptors and CARs, we made a series of anti-HER2 single-chain antibodies (scFvs; Fv denotes a heterodimer of the variable region of the light and heavy chains) with affinities that span a 100-fold range (dissociation constants between 2.0 and $200 \mathrm{nM}$ ) (Fig. 1D and fig. S2).

We constructed and tested several versions of the anti-HER2 synNotch $\rightarrow$ anti-HER2 CAR circuit in human primary CD8+ T cells in vitro (fig. S3A). To assay density-sensing behavior, we measured target cell killing by quantitative flow cytometry. Several circuit T cells showed antigen density ultrasensitivity [Hill coefficients

\footnotetext{
${ }^{1}$ Cell Design Institute, Department of Cellular and Molecular Pharmacology, University of California San Francisco, San Francisco, CA, USA. ${ }^{2}$ Center for Cellular Construction, University of California San Francisco, San Francisco, CA, USA. ${ }^{3}$ Department of Pharmaceutical Chemistry, Chan Zuckerberg BioHub, University of California San Francisco, San Francisco, CA, USA. ${ }^{4}$ Graduate Program in Bioengineering, University of California Berkeley and University of California San Francisco, San Francisco, CA, USA. ${ }^{5}$ Department of Virology, Haartman Institute, University of Helsinki, Helsinki, Finland.

*These authors contributed equally to this work

†Corresponding author. Email: wendell.lim@ucsf.edu
}

$\left(n_{\mathrm{H}}\right)$ of 1.7 to 4.4$]$ (Fig. 2A, bottom). By contrast, $\mathrm{T}$ cells with constitutive expression of either high- or low-affinity CARs showed little density discrimination (Fig. 2A, top). In circuit $\mathrm{T}$ cells in which a low-affinity synNotch receptor [scFv dissociation constant $\left.\left(K_{\mathrm{d}}\right)=210 \mathrm{nM}\right]$ was used to control the expression of a highaffinity CAR ( $\mathrm{scFv} K_{\mathrm{d}}=17.6 \mathrm{nM}$ ) (Fig. 2A, red line), their ultrasensitive threshold clearly discriminated between the target densities of $10^{4.5}$ and $10^{6.5}$ [Fig. 2B; $n_{\mathrm{H}}=4.4$, antigen den- sity that produces a $50 \%$ maximal response $\left(\right.$ den $\left._{50}\right)=10^{5.5}$ ] (for circuit activity in T cells from multiple donors, see fig. S4B).

The observed ultrasensitivity appears to originate from the designed transcriptional cascade. The steady-state amounts of CAR expression (monitored by tagging CARs with an mCherry protein) (fig. S3A) depended strongly on target antigen density (Fig. 2B, left, and fig. S4C, red line). $\mathrm{T}$ cell proliferation, a critical component of an antitumor response, also showed

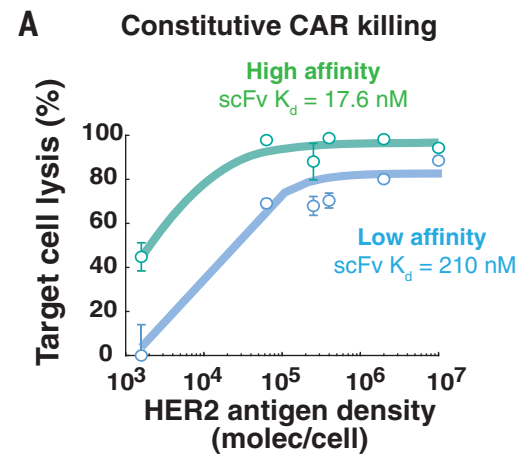

synNotch-CAR cascade killing

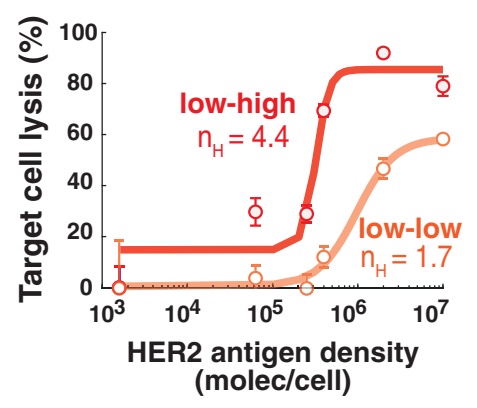

low-high scFv $\mathrm{K}_{\mathrm{d}}$ (210 nM synNotch, 17.6 nM CAR) low-low scFv $K_{d}$ (210 nM synNotch, 210 nM CAR)

Targets (HER2+ K562): 20,000

T cells (human primary CD8+): 10,000

Assay timepoint: 3 days

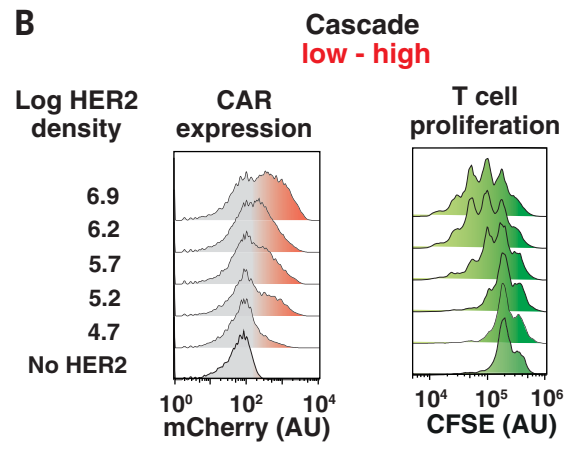

Circuit: Low affinity synNotch to high affinity CAR Targets (HER2+ K562): 20,000; T cells:10,000 Assay timepoint: 3 days

C
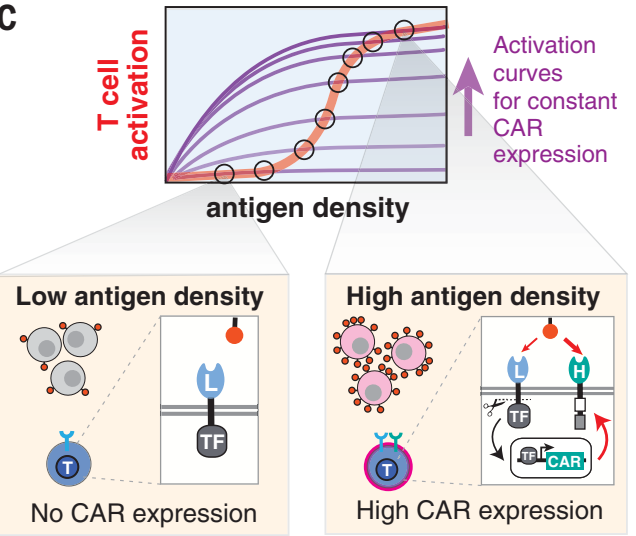

Fig. 2. A two-step low- to high-affinity recognition circuit yields ultrasensitive antigen-density sensing. (A) In vitro cell killing curves as a function of target cell antigen density, using human primary CD8+ T cells expressing a constitutive CAR of high ( $\left.s c F v K_{d}=17.6 \mathrm{nM}\right)$ or low affinity (scFv $K_{d}=210 \mathrm{nM}$ ) (top) or a two-step circuit in which the low-affinity synNotch receptor induces expression of either a low- or a highaffinity CAR (scFv $K_{d}=210 \mathrm{nM}$ synNotch, 17.6 nM CAR) (bottom). For the circuits, lines are fitted to a Hill equation (Hill coefficient for each curve is indicated) (fig. S4A). For constitutive CARs, the lines are drawn on the basis of inspection. The percentage of specific lysis was determined by using flow cytometry to count the number of target cells after 3 days relative to a coculture of targets in the presence of untransduced T cells (see fig. S3C for gating details). Data points denote means, and error bars represent SEM $(n=3)$. (B) Representative fluorescence-activated cell sorting (FACS) distributions $(n=3)$ for CAR expression and T cell proliferation measured as a function of target cell HER2 density (at 3 days) for $T$ cells expressing a low- to high-affinity recognition circuit. T cell proliferation was only observed at HER2 densities of $>10^{5}$ (fig. S4C). CFSE, carboxyfluorescein diacetate succinimidyl ester. (C) Model for the mechanism of a two-step circuit expressing a low-affinity synNotch to a high-affinity CAR. In principle, cells with this circuit display two very different responses; in the presence of a low-antigen-density target (left), the $T$ cell activity is dominated by the low-affinity synNotch and low amounts of a CAR. In the presence of a high-antigendensity target (right), the expression of a CAR is increased, and the T cell activity is dominated by the high-affinity CAR response that activates proliferation and killing. T cell activity is predicted to show a sigmoidal response curve (shown in red) because as antigen density increases, CAR expression also gradually increases, transiting between the series of linear response curves shown in purple. 
A

HER2 expression in target cancer cell lines

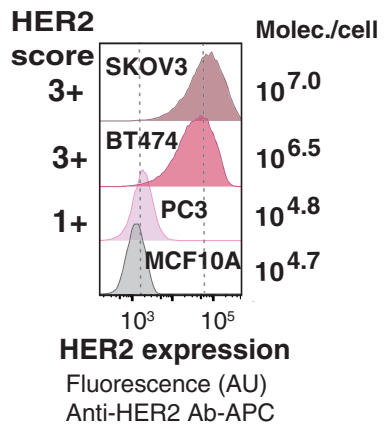

\section{B}

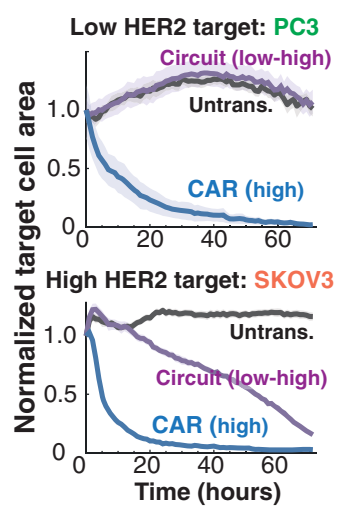

Cell killing in vitro

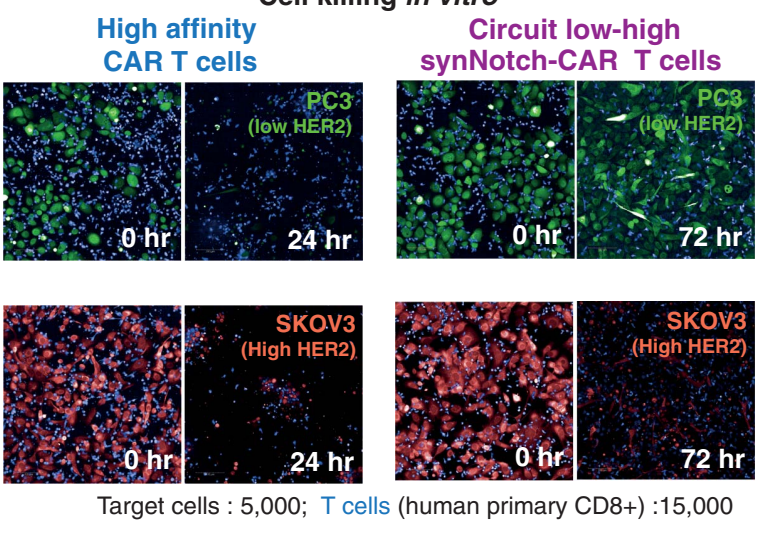

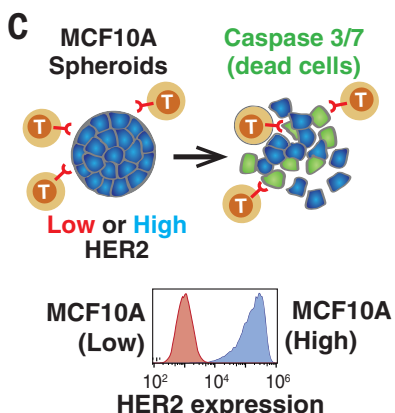

E

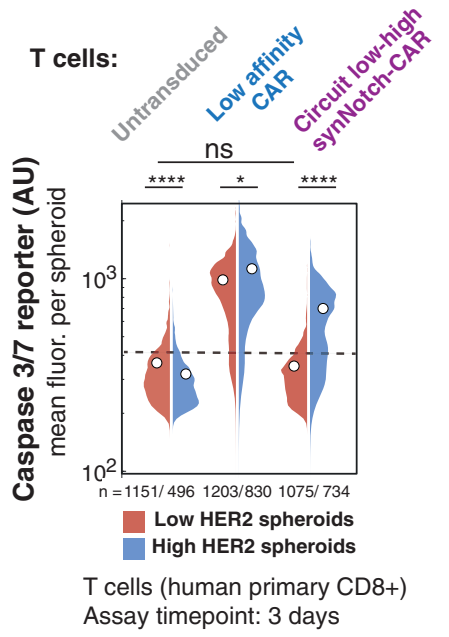

D

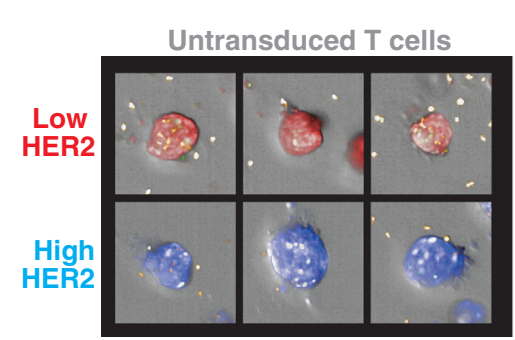

High vs Low HER2 spheroid killing

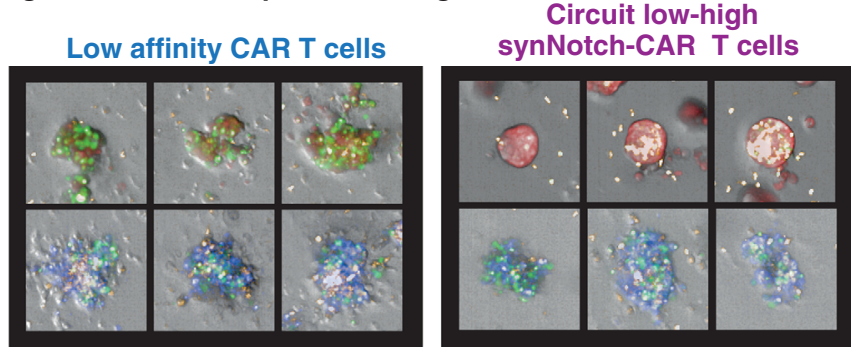

after 3 days co-culture; caspase 3/7 reporter
F Mixed high and low HER2 MCF10A spheroids

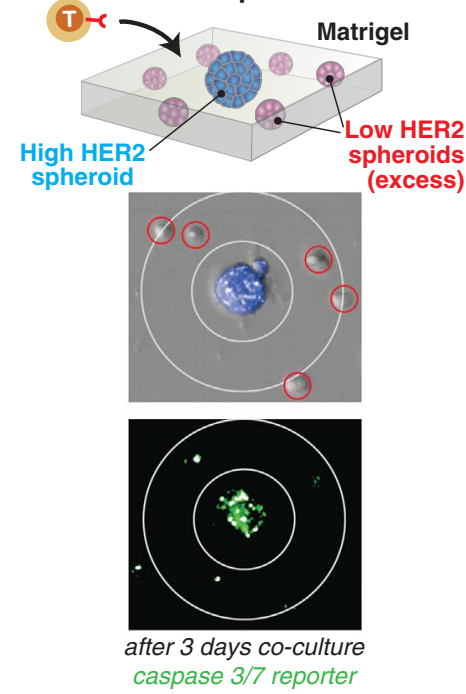

G

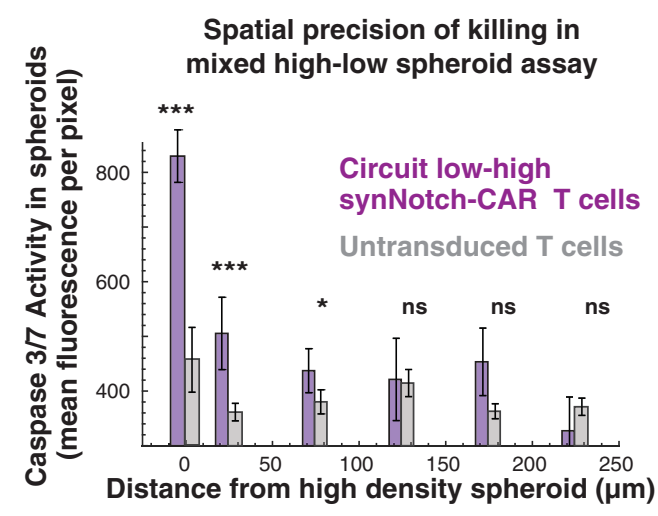

T cells (human primary CD8+) Assay timepoint: 3 days
Fig. 3. Low-to-high synNotch-to-CAR circuit: Discrimination between high- and low-density tumor cancer cell lines and 3D spheroids.

(A) Representative FACS distributions $(n=3)$ showing the HER2 expression of low- and high-HER2 cell lines. The HER2 score (as defined by ASCO-CAP scoring guidelines) is shown to the left, and the average HER2 density is shown to the right. (B) Area occupied by target cells as a function of time, normalized by the area occupied by target cells at time 0 (left). Low-HER2-density cancer cells (top plot), PC3 (1+ tumor line), or high-HER2-density cancer cells (bottom plot), SKOV3 (3+ tumor line), were cultured with human primary CD8+ $\mathrm{T}$ cells expressing either a two-step circuit low-affinity to high-affinity CAR (scFv $K_{d}=210 \mathrm{nM}$ synNotch, 17.6 nM CAR) (purple lines) or a high-affinity CAR $\left(\mathrm{scFv} K_{\mathrm{d}}=17.6 \mathrm{nM}\right)$ (blue lines). Gray lines correspond to the target area in the presence of untransduced T cells. Solid lines show the average normalized target area, and the shaded areas depict the SEM ( $n=3$ wells, three fields of view per well). To the right, representative images of the in vitro cell killing experiment are shown. T cells are shown in blue, the low-HER2-density cells in green, and the high-HER2-density cells in red (for data from additional cell lines, see fig. S6; see also movies S1 and S2). (C) Schematics of T cell killing assay of spheroids made of MCF10A cells expressing high or low HER2. A caspase dye (shown in green) was used to track cell death. The FACS distributions show the HER2 expression on MCF10A lines used to make the 3D spheroids. The MCF10A line engineered to express high HER2 is shown in blue, and wild-type MCF10As that express low levels of HER2 are shown in red. (D) Representative images of spheroids expressing low (shown in red) or high (shown in blue) HER2 in the presence of untransduced T cells (left) or T cell expressing either a low-affinity CAR (middle) or a two-step low-to-high 
recognition circuit (right). The caspase $3 / 7$ signal is in green, and the T cells are labeled in yellow. (E) Violin plots showing the distributions of mean caspase 3/7 signal per spheroid. The distributions for the low-HER2-density spheroids are shown to the left in red and the ones for the high-HER2-density spheroids to the right in blue. The mean of the distribution is shown as a white circle, and the number of analyzed spheroids in each case is shown at the bottom. The statistical significance of differences in mean caspase 3/7 signal in each coculture condition was determined by a Kruskal-Wallis test with Bonferroni's post hoc for multiple comparisons [not significant (ns) $>0.05$, ${ }^{*} P<0.05$, ****P $<0.0001]$. (F) Schematics of experiment to study the distance dependence of killing activity of low-to-high-circuit T cells in a 3D culture system. HighHER2-density spheroids were mixed with a large excess of low-HER2-density cells and engineered low-to-high-circuit T cells. A caspase 3/7 dye was used as a reporter for cell killing. The spheroids and cells were embedded in a thin slab of cell-laden Matrigel to constrain their position along the $z$ axis. A representative image of a high-HER2 spheroid is shown in blue surrounded by low-HER2 spheroids, highlighted in red circles, after 3 days of coculture. The corresponding image for the caspase 3/7 activity is shown below. (G) Caspase 3/7 activity (fluorescence per pixel within spheroids) is plotted as a function of distance from a high-HER2 spheroid, located at the origin. The distances for each low-density spheroid to the closest high-density spheroid were binned in 50- $\mu \mathrm{m}$ bins, and the means of the caspase signal of all spheroids within the bin were computed (on a per-pixel basis to account for differences in spheroid size). The gray bars show the mean values of caspase signal for spheroids cocultured with untransduced T cells; the purple bars show the mean values of caspase signal for spheroids cocultured with low-tohigh-circuit T cells. The error bars indicate the SEM. A two-sample KolmogorovSmirnov test was used to determine the significance of distributions differences (ns $>0.05,{ }^{*} P<0.05,{ }^{* * *} P<0.001$ ). For representative images for each channel showing high-density spheroids (blue) surrounded by low-HER2 spheroids, see fig. S7. T cells were labeled with a yellow cell trace dye. an ultrasensitive dependence on target antigen density (Fig. 2B, right, and fig. S4C, green line).

The response of the $\mathrm{T}$ cell circuit could be tuned by altering the receptor affinities within the circuit. For example, lowering the CAR affinity ( $\mathrm{scFv} K_{\mathrm{d}}=210 \mathrm{nM}$ ) while maintaining a low-affinity synNotch increased the density threshold $\left(\right.$ den $\left._{50}=10^{6.0}\right)$ (fig. S4D) but also reduced the maximal killing activity and lowered the overall ultrasensitivity $\left(n_{\mathrm{H}}=1.7\right)$. The ultrasensitivity began to break down when we used a medium-affinity synNotch ( $\mathrm{scFv} K_{\mathrm{d}}=$ $46.5 \mathrm{nM}$ ) receptor as the circuit filter (fig. S4D, brown line). Thus, a robust ultrasensitive response is generated by linking low- and highaffinity recognition into a two-step cascade (Fig. 2C). Low-affinity synNotch receptors that encounter low densities of HER2 antigen produce low amounts of CAR expressed at the $\mathrm{T}$ cell surface (Fig. 2C, left). By contrast, when low-affinity synNotch receptors encounter high HER2 densities (Fig. 2C, right), they express higher steady-state amounts of CARs. Thus, high target antigen density both increases CAR expression level and activates $\mathrm{T}$ cell proliferation and killing, leading to a nonlinear all-or-none response (Fig. 2C).

By contrast, we found that constitutively expressed CARs, even with reduced CAR expression or affinity (fig. S3B), triggered T cell killing of low-density antigen cells (fig. S5, A and B). CARs and synNotch receptors showed different sensitivities, even when using the same antigen binding domain. CARs are more sensitive, triggering killing activity with relatively low antigen density (Fig. 2A and fig. S5), whereas synNotch-mediated gene expression requires higher antigen densities. Thus, the low-affinity synNotch receptor does not induce a sufficient amount of CAR expression to trigger a killing response with low-density targets and only initiates strong killing with high-density targets [Fig. 2, A (bottom) and $\mathrm{B}$, and fig. $\mathrm{S} 4 \mathrm{C}]$.

We further tested the synNotch ${ }_{\text {low affinity }} \rightarrow$ $\mathrm{CAR}_{\text {high affinity }}\left(\mathrm{scFv} K_{\mathrm{d}}=210 \mathrm{nM}\right.$ synNotch, 17.6 nM CAR) HER2 antigen density-sensing circuit against a number of different human cancer cell lines with low and high HER2 expression (Fig. 3, A and B, and fig. S6). In in vitro killing assays, engineered $\mathrm{T}$ cells were mixed with target cancer cells that expressed either low-density HER2 [human prostate adenocarcinoma (PC3), $10^{4.8}$ HER2 molecules per cell, HER2 score 1+] or high-density HER2 [human ovary adenocarcinoma (SKOV3), $10^{7.0}$ HER2 molecules per cell, HER2 score $3+]$. With the low-HER2-density cells, neither the untrans-

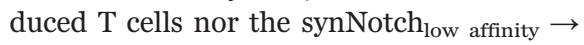
$\mathrm{CAR}_{\text {high affinity }}$ circuit $\mathrm{T}$ cells showed cytotoxicity over 72 hours (Fig. 3B and movie S2). For the high-HER2-density cells, the synNotch ${ }_{\text {low affinity }} \rightarrow$ $\mathrm{CAR}_{\text {high affinity }}$ circuit $\mathrm{T}$ cells effectively killed the high-HER2 cells (movie S2). However, we observed delayed activation onset and a lag in the time required for complete killing compared with the conditions observed for $\mathrm{T}$ cells with constitutive CAR expression (72 hours versus 24 hours) (movie S1). This delay is consistent with a model in which CAR expression mediated by synNotch recognition requires additional time to accumulate sufficient CAR for effective killing (Fig. 3B). Similar discriminatory behavior was observed against other human cancer lines of varying HER2 densities (fig. S6, A and B). By contrast, the constitutive CAR-T cells (both high- and low-affinity CARs) rapidly eliminated both the low- and high-HER2 target cells in this assay (figs. S5D and $\mathrm{S} 6, \mathrm{~A}$ and $\mathrm{B}$, and movie S1). In the circuit $\mathrm{T}$ cells, CAR expression and $\mathrm{T}$ cell proliferation also showed a clear dependence on the input HER2 antigen density (fig. S6C, top row). The synNotch low affinity $\rightarrow \mathrm{CAR}_{\text {high affinity }} \mathrm{T}$ cells notably improve discrimination between multiple high- and low-density cancer cells, but the timing of circuit activation results in the delayed onset of tumor killing.

To evaluate how these ultrasensitive densitysensing $\mathrm{T}$ cells behave in a more complex multicellular context, we used a three-dimensional (3D) target spheroid culture model $(27,28)$. We engineered a human breast epithelial MCF10A line, which normally expresses low amounts of
HER2 $\left(10^{4.7}\right.$ HER2 molecules per cell, HER2 score 0 ), to express high HER2 (equivalent to HER2 score 3+) (Fig. 3C). We assembled 3D spheroids (29) using either low- or high-HER2 MCF10A cells and embedded them in Matrigel with engineered T cells. Using 3D confocal microscopy and a caspase activity dye, we quantified the caspase fluorescence per spheroid over 3 days as an assay for target cell killing (Fig. 3C). The two-step circuit synNotch low affinity $\rightarrow$ $\mathrm{CAR}_{\text {high affinity }}\left(\mathrm{scFv} K_{\mathrm{d}}=210 \mathrm{nM}\right.$ synNotch, 17.6 nM CAR) T cells effectively invaded, killed, and disassembled the high-HER2-density spheroids but discriminated against the lowHER2 spheroids (Fig. 3, D and E, right). By contrast, a low-affinity anti-HER2 CAR killed and disassembled the low- and high-HER2-density spheroids indiscriminately (Fig. 3, D and E, middle).

We also used this spheroid assay to evaluate the degree of spatial discrimination. If a circuit $\mathrm{T}$ cell was activated by a high-density spheroid, could it then migrate to a low-density spheroid and kill those cells? We mixed a low number of high-HER2 spheroids with an excess of lowHER2 spheroids and T cells, all embedded in Matrigel. T cells were observed to migrate freely among the spheroids (Fig. 3F and fig. S7, B and C). We then measured the caspase signal within the low-HER2 spheroids as a function of radial distance from the closest high-HER2 spheroid (Fig. 3F). We found a very sharp decay of killing activity as a function of distance from the high-HER2 spheroid and estimated a radius of off-target killing to be $<100 \mu \mathrm{m}$ (Fig. $3 \mathrm{G})$. The circuit $\mathrm{T}$ cells only infiltrated, expanded, and launched a killing response in high-antigen-density spheroids (fig. S7, B and C). Many aspects of $\mathrm{T}$ cell activation, including increased adhesion and production of local cytokine gradients, may contribute to this high level of spatial discrimination.

Finally, we evaluated the density discrimination of synNotch ${ }_{\text {low affinity }} \rightarrow \mathrm{CAR}_{\text {high affinity }}$ ( $\mathrm{scFv} K_{\mathrm{d}} 210 \mathrm{nM}$ synNotch, $17.6 \mathrm{nM}$ CAR) circuit T cells in multiple mouse tumor models (Fig. 4A). We first implanted immunocompromised NOD scid gamma (NSG) mice 
A

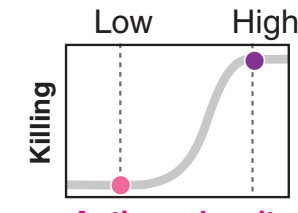

Antigen density

Two tumor mouse model

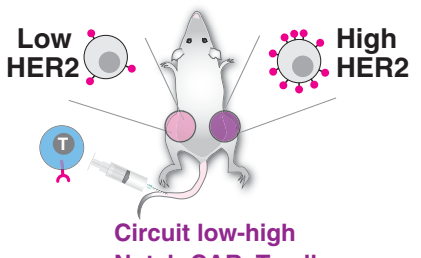

synNotch-CAR T cells

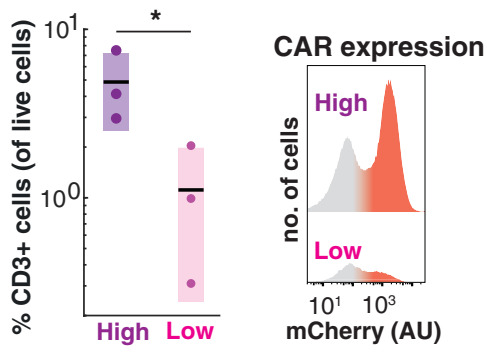

Recovered T cells from single tumor mice Assay timepoint: 7 days post $\mathrm{T}$ cell injection

D

Circuit T cell distribution in dual tumor mouse model

Two tumor mouse model

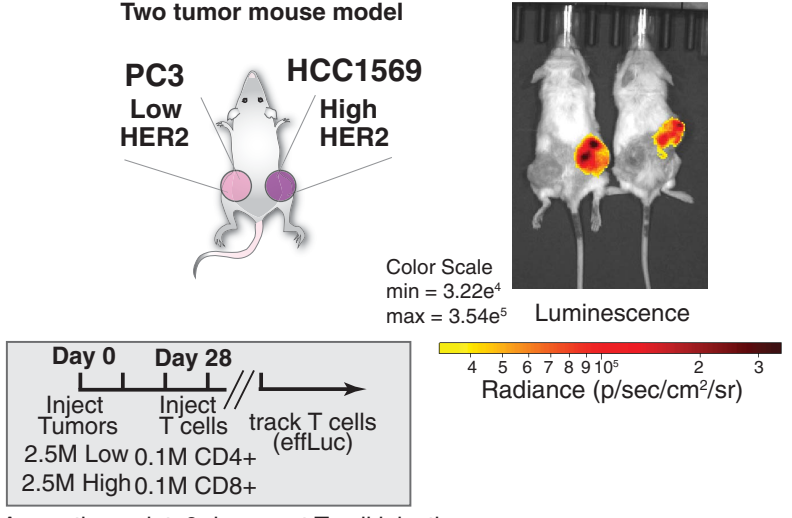

Assay timepoint: 9 days post $\mathrm{T}$ cell injection

Fig. 4. Low-o-high synNotch-to-CAR circuit: Antigen density discrimination in mouse models. (A) Schematics of a two-tumor mouse model experiment to test the efficacy and safety of ultrasensitive antigen density-sensing T cells: low-affinity synNotch to high-affinity CAR circuit (scFv $K_{d}=210 \mathrm{nM}$ synNotch, $17.6 \mathrm{nM}$ CAR). Low- and high-HER2 tumor cells were injected subcutaneously in the flanks of NSG mice. Engineered primary human CD4+ and CD8+ T cells were injected intravenously at the indicated times after tumor injection. Tumor volume was monitored through caliper measurement over several days after tumor injection. (B) FACS distributions showing the HER2 expression of cell lines used in the experiment. The doses and injection times for tumors and T cells are indicated in the gray box. Tumor volumes of cells with high and low K562 HER2 density after treatment with T cells expressing a two-step circuit (low-affinity synNotch to high-affinity CAR) are shown. The high-density tumor is shown in dark purple and the low-density tumor in pink. The solid lines connect the means, and the error bars indicate the SEM $(n=7)$. The gray and black dotted lines show the low-density and high-density tumor volumes after treatment with untransduced $\mathrm{T}$ cells, respectively (for details of control experiment, see fig. S10A). UnT, untransduced.
B

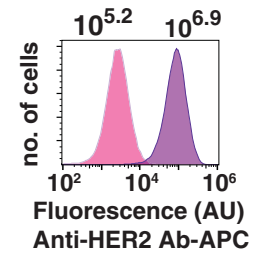

Day $0 \quad$ Day 7

$\underset{\text { nject Inject }}{\stackrel{\mathrm{H}}{\mathrm{Tumor}}}$

Tumors Trells measurement

5M Low 4M CD4+ via caliper

$5 \mathrm{M}$ High 4M CD8+

$\mathbf{E}$

PC3 SKOV3 $10^{4.8} \quad 10^{7.0}$

HER2 K562 tumors: High vs Low

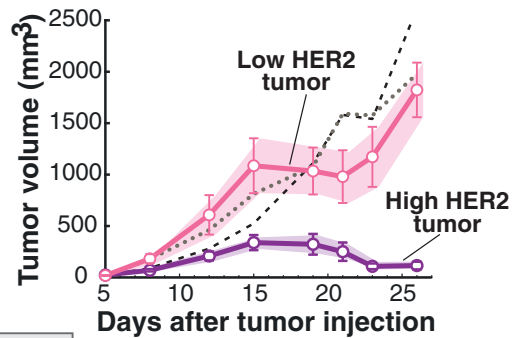

Days after tumor injection

\begin{tabular}{llc}
$\begin{array}{c}\text { Longitudinal } \\
\text { Comparison }\end{array}$ & & p-value (adjusted) \\
${$\cline { 1 - 2 }$} }$ & ns, $0.2518(0.755)$ \\
High: Circuit vs UnT & $0.0002(0.0013)$
\end{tabular}

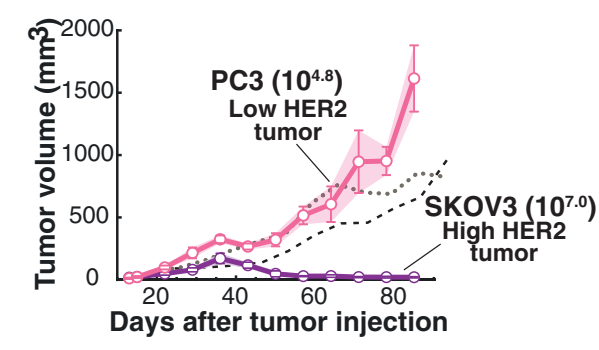

\begin{tabular}{|c|c|c|}
\hline Day $0 \quad$ Day 28 & $\begin{array}{l}\text { Longitudinal } \\
\text { Comparison }\end{array}$ & $\mathrm{p}$-value (adjusted) \\
\hline $\begin{array}{l}\text { Tumors T cells measurement } \\
\text { 1.0M Low 1M CD4+ via caliper } \\
\text { 1.5M High 1M CD8+ }\end{array}$ & $\begin{array}{l}\text { Low: Circuit vs UnT } \\
\text { High: Circuit vs UnT }\end{array}$ & $\begin{array}{l}\text { ns, } 0.856(1.0) \\
<0.0001 \quad(<0.0001)\end{array}$ \\
\hline
\end{tabular}

F MDA-231 HCC1569

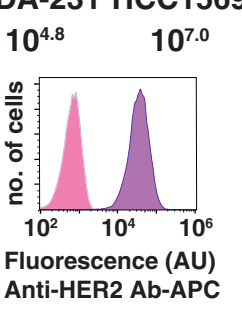

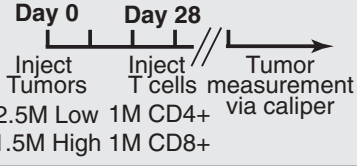

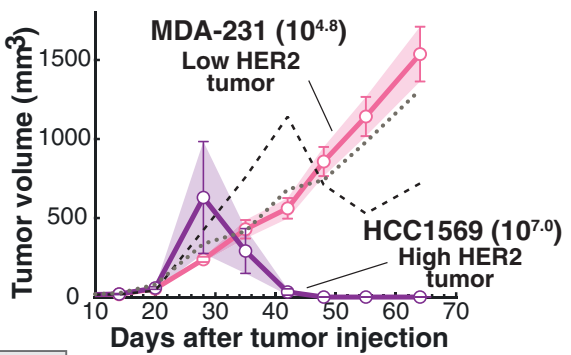

Longitudinal

\begin{tabular}{lcc} 
Comparison & & $p$-value (adjusted) \\
\cline { 1 - 1 } Low: Circuit vs UnT & ns, $0.232(0.485)$ \\
High: Circuit vs UnT & $0.0018(0.0106)$
\end{tabular}

(C) Fraction of CD3+ T cells infiltrated in high or low K562 tumors 7 days after T cell injection. Representative FACS distributions $(n=3)$ showing the CAR expression (mCherry tagged) in CD3+ engineered T cells are given. (D) Schematics of a dualtumor mouse model to test the circuit $T$ cell distribution. The doses and injection times for tumors and $T$ cells are indicated in the gray box. A representative image of luciferase activity in dual-tumor mice treated with low-to-high-circuit T cells 9 days after T cell injection is shown. Luciferase signal was only detected in the high-HER2 tumor, indicating localized expansion $(n=2)$. effLuc, firefly luciferase. (E) Tumor volumes of cancer lines PC3 (low) and SKOV3 (high) after treatment with T cells expressing a two-step circuit (low-affinity synNotch to high-affinity CAR) $(n=5)$. The doses and injection times for tumors and $\mathrm{T}$ cells are indicated in the gray box. (F) Tumor volumes of cancer lines MDA-231 (low) and HCC1569 (high) after treatment with $T$ cells expressing a two-step circuit (low-affinity synNotch to high-affinity CAR) $(n=6)$. The doses and injection times for tumors and T cells are indicated in the gray box. For more details and individual mouse tumor volume plots, see fig. S9. Statistical longitudinal analyses were performed over entire segments of the tumor growth curves by using TumGrowth (32). See materials and methods for more details. 
with a high-HER2-density K562 tumor on one side and a low-HER2-density K562 tumor on the opposite side. After establishing the tumors, we injected the tail vein with a mix of equal numbers of CD4+ and CD8+ primary human T cells transduced with the synNotch low affinity $_{\rightarrow}$ $\mathrm{CAR}_{\text {high affinity }}$ circuit. The circuit $\mathrm{T}$ cells showed strong density discrimination (Fig. 4B and fig. S9A); the high-density tumors were cleared rapidly, but the low-density tumors grew at similar rates to those observed for untransduced $\mathrm{T}$ cells. This tumor discrimination was also observed at a fivefold higher effector-totarget ratio (fig. S12). The circuit $\mathrm{T}$ cells show significant expansion and induced CAR expression only within the high-density tumors (Fig. 4, C and D, and fig. S11). Consistent discrimination was observed with other pairs of human cancer cell lines that expressed high $\left(\sim 10^{7.0}\right.$ molecules per cell $)$ or low $\left(\sim 10^{5}\right.$ molecules per cell) amounts of HER2 (Fig. 4, E and F, and fig. S9, B and C). We performed control experiments with untransduced T cells (fig. S10) or $\mathrm{T}$ cells constitutively expressing either a lowor a high-affinity anti-HER2 CAR (fig. S8). The constitutive low- or high-affinity CAR-T cells showed poor density discrimination, clearing both the low- and high-density tumors (fig. S8).

This work demonstrates that a general design principle, the use of a two-step regulatory circuit to generate an ultrasensitive doseresponse behavior, can be used to engineer $\mathrm{T}$ cells that discriminate between target cells with high- and low-antigen-density expression. These two-step synNotch-to-CAR circuits function well both in vitro and in vivo, and the threshold can be tuned by altering the affinities of the synNotch and CAR receptors. This approach should enable expansion of the repertoire of target antigens to include other examples that are overexpressed in cancer cells compared with normal cells. Indeed, we were able to show that engineered $\mathrm{T}$ cells with a low-affinity synNotch receptor to a highaffinity CAR circuit, built from anti-EGFR binding domains $(30,31)$, resulted in ultrasensitive EGFR density-sensing T cells (fig. S13).

The effective deployment of therapeutic $\mathrm{T}$ cells to treat solid tumors will require overcoming several other challenges, including tumor heterogeneity and suppressive tumor microenvironments, as well as improving trafficking of cells to the tumors. However, the ability to achieve ultrasensitive antigen-density discrimination provides a critical tool for widening the therapeutic window of engineered T cells against solid cancers, in which many tumor-associated antigens are overexpressed but not absolutely tumor specific.

\section{REFERENCES AND NOTES}

1. W. A. Lim, C. H. June, Cell 168, 724-740 (2017)

2. L. M. Whilding, J. Maher, Mol. Oncol. 9, 1994-2018 (2015).

3. J. N. Brudno, J. N. Kochenderfer, Nat. Rev. Clin. Oncol. 15 31-46 (2018)

4. M. M. D’Aloia, I. G. Zizzari, B. Sacchetti, L. Pierelli, M. Alimandi, Cell Death Dis. 9, 282 (2018).

5. S. L. Maude et al., N. Engl. J. Med. 378, 439-448 (2018).

6. S. S. Neelapu et al., N. Engl. J. Med. 377, 2531-2544 (2017).

7. S. J. Schuster et al., N. Engl. J. Med. 380, 45-56 (2019).

8. R. A. Morgan et al., Mol. Ther. 18, 843-851 (2010).

9. C. H. Lamers et al., Mol. Ther. 21, 904-912 (2013).

10. F. C. Thistlethwaite et al., Cancer Immunol. Immunother. 66 1425-1436 (2017).

11. A. McCabe, M. Dolled-Filhart, R. L. Camp, D. L. Rimm, J. Natl. Cancer Inst. 97, 1808-1815 (2005).

12. W. Yasui et al., Cancer Res. 48, 137-141 (1988).

13. M. R. Parkhurst et al., Mol. Ther. 19, 620-626 (2011).

14. Y. Guo et al., Clin. Cancer Res. 24, 1277-1286 (2018).

15. Q. Zhang, S. Bhattacharya, M. E. Andersen, Open Biol. 3 , 130031 (2013)

16. S. A. Frank, Biol. Direct 8, 31 (2013).

17. S. Hooshangi, S. Thiberge, R. Weiss, Proc. Natl. Acad. Sci. U.S.A. 102, 3581-3586 (2005).

18. J. E. Ferrell Jr., S. H. Ha, Trends Biochem. Sci. 39, 612-618 (2014)

19. X. Wan et al., Nat. Chem. Biol. 15, 540-548 (2019).

20. C. Y. Huang, J. E. Ferrell Jr., Proc. Natl. Acad. Sci. U.S.A. 93, 10078-10083 (1996).

21. D. Busse et al., Proc. Natl. Acad. Sci. U.S.A. 107, 3058-3063 (2010).

22. C. Y. Wu, L. J. Rupp, K. T. Roybal, W. A. Lim, Curr. Opin. Immunol. 35, 123-130 (2015)

23. K. T. Roybal et al., Cell 167, 419-432.e16 (2016)

24. K. T. Roybal et al., Cell 164, 770-779 (2016).

25. L. Morsut et al., Cell 164, 780-791 (2016).
26. M. Klichinsky et al., Nat. Biotechnol. 38, 947-953 (2020)

27. V. Dangles-Marie et al., Cancer Res. 63, 3682-3687 (2003).

28. M. Pickl, C. H. Ries, Oncogene 28, 461-468 (2009),

29. A. E. Cerchiari et al., Proc. NatI. Acad. Sci. U.S.A. 112 , 2287-2292 (2015)

30. A. Lehmann et al., mAbs 7, 1058-1071 (2015).

31. R. C. Roovers et al., Int. J. Cancer 129, 2013-2024 (2011).

32. D. P. Enot, E. Vacchelli, N. Jacquelot, L. Zitvogel, G. Kroemer, Oncolmmunology 7, e1462431 (2018).

\section{ACKNOWLEDGMENTS}

We thank K. T. Roybal, A. Ng, and G. Allen for sharing DNA plasmids: M. Moasser for sharing tumor cell lines for in vivo experiments; W. McKeithan for assistance with microscopy data collection; I. Eigl for assistance with biolayer interferometry data collection; A. Li, J. Choe, and members of the Lim Lab for advice and helpful discussions; and V. Nguyen and G. Allen for critical reading of this manuscript. Funding: This work was supported by NIH grants (P50GM081879, U54CA244438, and R01 CA196277 to W.A.L.), Howard Hughes Medical Institute (W.A.L.), and the UCSF Center for Cellular Construction (DBI-1548297), an NSF Science and Technology Center. R.A.H.-L. is a Cancer Research Institute Irvington Fellow supported by the Cancer Research Institute. R.A.H.-L. was a postdoctoral fellow of UC-MEXUS. Author contributions: R.A.H.-L. and W.A.L. conceived the project. The experimental plan was implemented by R.A.H.-L., W.Y., K.A.C. O.A.C., M.d.P.L.P., Y.T., and A.D.G.; R.A.H.-L., K.A.C., and O.A.C. designed and carried out 3D culture experiments supervised by Z.J.G. and W.A.L.; R.A.H.-L., W.Y., Y.T., and A.D.G. designed and carried out in vivo experiments supervised by W.A.L.; and A.M. and K.S. designed protein expression vectors and provided SCFv-GST purified protein constructs. R.A.H.-L. analyzed all data. R.A.H.-L. and W.A.L. prepared the figures and wrote the manuscript with suggestions from all authors. W.A.L. supervised all aspects of the work. Competing interests: A provisional patent application has been filed by the University of California related to this work (U.S. application number 62738995). Z.J.G. is an equity holder in Scribe Biosciences and Provenance Bio. Data and materials availability: All data are available in the manuscript or supplementary materials. Reagents are available from the corresponding author upon reasonable request. Plasmids from this paper will be made available on Addgene (deposit 79031).

\section{SUPPLEMENTARY MATERIALS}

science.sciencemag.org/content/371/6534/1166/suppl/DC1 Materials and Methods

Figs. S1 to S13

References

MDAR Reproducibility Checklist

Movies S1 and S2

View/request a protocol for this paper from Bio-protocol.

10 April 2020; accepted 11 February 2021

Published online 25 February 2021

10.1126/science.abc1855 


\section{Science}

\section{T cell circuits that sense antigen density with an ultrasensitive threshold}

Rogelio A. Hernandez-Lopez, Wei Yu, Katelyn A. Cabral, Olivia A. Creasey, Maria del Pilar Lopez Pazmino, Yurie Tonai, Arsenia De Guzman, Anna Mäkelä, Kalle Saksela, Zev J. Gartner and Wendell A. Lim

Science 371 (6534), 1166-1171.

DOI: 10.1126/science.abc1855originally published online February 25, 2021

\section{Designing smarter anticancer $\mathrm{T}$ cells}

Biological signaling systems can exhibit a large, nonlinear--or "ultrasensitive"--response, which would be useful to engineer into therapeutic T cells to allow for better discrimination between cancer cells and normal tissues. Hernandez-Lopez et al. modified human T cells using a two-step mechanism that allowed them to kill cells expressing large amounts of cancer marker protein but not cells expressing a small amount of the same protein. A first synthetic receptor recognized the antigen with low affinity. That receptor signaled to increase expression of a chimeric antigen receptor (CAR) with high affinity for the same antigen. The circuit proved effective in cell culture and mouse cancer models, offering hope of extending the CAR T cell strategy against solid tumors.

Science, this issue p. 1166

ARTICLE TOOLS

SUPPLEMENTARY

MATERIALS

RELATED
CONTENT

REFERENCES

PERMISSIONS http://science.sciencemag.org/content/371/6534/1166

http://science.sciencemag.org/content/suppl/2021/02/24/science.abc1855.DC1 http://stm.sciencemag.org/content/scitransmed/12/571/eaaz6667.full http://stm.sciencemag.org/content/scitransmed/13/578/eaba7308.full http://stm.sciencemag.org/content/scitransmed/12/571/eabd6169.full http://stm.sciencemag.org/content/scitransmed/13/575/eabb6295.full

This article cites 32 articles, 7 of which you can access for free http://science.sciencemag.org/content/371/6534/1166\#BIBL

http://www.sciencemag.org/help/reprints-and-permissions

Use of this article is subject to the Terms of Service

Science (print ISSN 0036-8075; online ISSN 1095-9203) is published by the American Association for the Advancement of Science, 1200 New York Avenue NW, Washington, DC 20005. The title Science is a registered trademark of AAAS.

Copyright (C) 2021 The Authors, some rights reserved; exclusive licensee American Association for the Advancement of Science. No claim to original U.S. Government Works 